\title{
Protein networks in diseases
}

\author{
Kayarkar N. A. ${ }^{1}$, Durgude S. G. ${ }^{1}$, Maurya B.D. ${ }^{1}$, Pawar S. V. ${ }^{1}$, Chate P. B. ${ }^{2}$ \\ ${ }^{1}$ V.E.S. College of Arts, Science and Commerce, Chembur, Mumbai-400 071, India \\ ${ }^{2}$ Bhavan's College, Andheri (west), Mumbai-400 058, India
}

\begin{abstract}
Protein interactions and their specificity play important roles in protein structure and functions. Identification and characterization of protein interfaces is thus important for the study. Adhesive interactions can be considered as one of the key features of many interactions. Computational approach can be applied to great extent in identifying disease specific genes. For instance, Nfat and tat proteins have specific roles related to diseases whose roles can be studied. Apoptosis is considered as a crucial part of many cellular activities and is shown to be related to proteolysis, inflammatory responses, in cancer perturbed protein interaction and mitochondrial dynamics.
\end{abstract}

Keywords- Protein interactions, apoptotic proteins, autophagy proteins, misfolding, inflammatory therapies

\section{Introduction}

Proteins are organic compounds made of amino acids arranged in a linear chain to form form a polymer. Protein-protein interactions involve not only the direct-contact association of protein molecules but also longer range interactions through the electrolyte, aqueous solution medium surrounding neighbor hydrated proteins over distances from less than one nanometer to distances of several tens of nanometers. Protein associations are also studied from the perspectives of biochemistry, quantum chemistry, molecular dynamics, signal transduction and other metabolic or genetic/epigenetic networks. The interactions between proteins are important for numerous biological functions. For example, mediating signals from the exterior to the inside of the cell by protein-protein interactions of the signaling molecules. This process, called signal transduction, plays a basic role in many biological processes and in many diseases (e.g. cancers). Proteins might interact for a long time to form part of a protein complex, a protein may be carrying another protein (for example, from cytoplasm to nucleus or vice versa in the case of the nuclear pore importins), or a protein may interact briefly with another protein just to modify it (for example, a protein kinase will add a phosphate to a target protein). Biological processes are not realized by a single molecule, but rather by the complex interaction of proteins with their environment, including nucleic acids, ions, lipids, membranes and, of course, other proteins. Thus, while the analysis of the structure and function of individual proteins is crucial for the understanding of their role in biological processes, it has a limited capability to explain the processes themselves. Some diseases are caused by simple genomic events that mutate or eliminate specific genes (e.g. frame shift mutations or insertion of viral genes). On the other hand, there are "complex diseases", such as heart or psychiatric diseases that are multifactorial. Between these two ends lie many, if not most, of the pathologies and illnesses, including AIDS, cancer, and Alzheimer's disease.

\section{Protein Therapeutics}

Protein therapeutics involve medical treatment that has wide-reaching therapeutic possibilities and applications currently being developed in many fields like Cancer,-Diabetes, Liver disease, Brain disease. It can also be applied in immunologic and inflammatory diseases. The immune system is a complex and highly developed system, and its function is to search for and kill invaders. If a person is born with a severely defective immune system, death from infection by a virus, bacterium, fungus or parasite will occur. Inflammation is a protective attempt by the organism to remove the injurious stimuli as well as initiate the healing process for the tissue. Even in cases where inflammation is caused by infection, the two are not synonymous: infection is caused by an exogenous pathogen, while inflammation is the response of the organism to the pathogen. Hence protein therapeutics plays a very important role in treating and preventing such diseased conditions.

\section{Protein Interactions and Diseases}

Protein interactions play an important role in their structure and function. The macromolecular assemblies are stabilized by specific non covalent interactions that allow proteins to form complexes. Some structure based computational methods are developed to characterize the quaternary structure of oligomeric proteins and analyze the properties of interfaces between protein subunits. The size, chemical and amino acid composition and atomic packing of subunit interfaces of protein complex are compared and a distinction can be made [1]. A study was done investigating the types of interactions specific to the biological function, and types of interactions which are persistent regardless of their function in transient protein-protein heterocomplexes. A systematic analysis was thus performed using canonical and noncanonical interactions to represent the physicochemical properties of protein-protein interfaces. On analyzing the interfaces of transient protein-protein heterocomplexes they showed specific 
interaction types based on their functional category and such interaction types were conserved through the common binding mechanism. Understanding protein interaction is thus an important challenge. Computational research on protein interaction has helped in prediction and understanding nature of interactions and their 3-D structure [2, 3]. The biological process in cells is mainly governed by protein interactions. Since protein interfaces are sites where protein interact physically, their identification and characterization helps in identifying protein interactions. Interface predictions can be done using methods which use structural and physico-chemical characteristics that distinguish interface residues from non-interface surface residues. Further, protein-protein interfaces were extracted from a 3-D structural data of protein complexes and then clustered to derive biological insights. Since conventional protein interface clustering methods lack computational skills and statistical assistance, a new method "PPiClust" showing above features was presented. The interface clusters can thus help in uncovering the structural basis of protein interactions. The analysis revealed diverse protein groups with similar interface patterns and some showed presence of linear binding motifs.

The development of high-throughput technologies has also proved to produce several large scale protein interaction data sets for multiple species. The basic units of protein interactions are domain interactions and it is vital to understand protein interactions at the level of the domains. The exposure and accuracy of predicted domain interactions can be significantly increased by integrating multiple data sources [46]. In a particular research, it was studied that the Herpesviruses make up a family of large DNA viruses widely spread in vertebrates, causing a variety of different diseases. Further, on studying three additional herpesvirus species, herpes simplex virus 1 (HSV-1), murine cytomegalovirus and Epstein-Barr virus, a core set of highly conserved protein interactions were identified. Interactions are conserved between orthologous proteins despite low sequence similarity, suggesting that function may be more conserved than the sequence [7]. While another study revealed, the inducible form of cyclooxygenase is COX-2 expression, whose tight regulation is necessary for controlling eicosanoid production in atherosclerosis and other inflammatory syndromes. Adhesive interactions between platelets and monocytes occur in these conditions and deliver specific signals that trigger inflammatory gene expression. In monocytes, activated platelets induce COX-2 synthesis by combinatorial signaling to transcriptional and posttranscriptional checkpoints which can be changed [8].

\section{Protein Networks in Diseases}

Network topology has been analyzed to study biochemical networks on a large scale to increase network robustness. Many biological processes involve protein networks. Reports indicated a balance between activating and inhibiting connections is important in determining whether network dynamics reach steady state or oscillate. A model was used to study the random networks, networks selected for robust dynamics and biological network topologies. In addition to this, Genomic transcriptional profiles were reconstructed by a computational approach on adult human tissues. The information was obtained from unbiased UniGene cDNA libraries. Distribution of gene expression levels in a tissue thus appears to follow a power law, that signifies a correspondence between transcriptional profile and scale-free topology of protein network. The expressions were analyzed and compared with reference human genes. Disease genes were more expressed than expected which suggested a possible correspondence of their products to important nodes of intra cellular protein network $[9,10]$. Pulmonary surfactant proteins and lipids are essential after birth for normal functioning of lungs. Lung immaturity and surfactant deficiency causes respiratory distress syndrome observed in infants. In late gestation, fetal lung undergoes maturation which adapts it to air breathing at birth. The study indicated Calcineurin $(\mathrm{Cn})$ is important in controlling such mechanisms of perinatal lung maturation. Whereas, nuclear factor of activated T cells (Nfat) was identified to be the direct activator in adapting to air breathing [11]. Human immunodeficiency virus (HIV) associated dementia (HAD) is a neuropsychiatric disorder. Since HIV-I does not infect neurons, HIV-I neurotoxicity is indirect resulting from the release of HIV transactivator of transcription (Tat). Virus infected cells release Tat. Effects of Tat on synapses between hippocampal neurons causes synapse loss and cell death. A method was thus invented for generating a network of direct and indirect interaction partners of a disease related polypeptide. The method also relates to a protein complex comprising of atleast 2 proteins and to methods for identifying compounds involved in it [12]. The identification of key target nodes within complex molecular networks is significant since the results of pathway analysis are usually sets of fairly complex networks or functional processes. Computational methodology is useful in predicting a lot of key regulatory genes and proteins in disease- and condition-specific biological networks. It has great potential for developing network-based combinational treatment strategies for a wide range of diseases. An application of computational methods can be seen in Protein kinases that control cellular decision processes by phosphorylating specific 
substrates. Since proteome-wide mapping has identified thousands of in vivo phosphorylation sites, a computational method, NetworKIN, can augment motifs with context for kinases and phosphoproteins and yields a 2.5-fold improvement in the accuracy which can be used in constructing phosphorylation networks [13, 14].

\section{Proteins in Apoptosis and Autophagy}

Apoptosis can either inhibit or promote other cells and their activities. Autophagy plays an important role in immunity and pathogenesis of diseases and also shows stimulation by inactivation of p53. Protein misfolding can lead to cancer showing various roles in lung cells, kallikrein formation. Amyloid formation is also known to be associated to it. Unfolded protein accumulation can lead to B-cell lymphoiesis. Intrinsically disordered proteins are found to be involved in colon cancer. Conformational changes in amyloidogenic proteins leads to aggregation of proteins. Protein misfolding can trigger Alzheimer's disease while expansion of the polyglutamine tract in the huntingtin (Htt) protein, can lead to Huntington's disease. Mammalian prion protein (PrP) is prevented from translocation during endoplasmic reticulum stress leading to neurodegeneration. Protein therapeutics for immunologic diseases includes use of intravenous Ig, peptide antigens for autoimmune and allergic disorders and therapeutic capacity of regulatory $T$ cells. Junctional adhesion molecule-C (JAM-C) plays an important role in arthritis. Also IL-32 is closely associated with TNFa, and contributes to the exacerbation of TNFa-related inflammatory arthritis and colitis. Apoptosis or programmed cell death is a morphologically distinct form of cell death. It is very crucial for many processes of a cell like normal cell turn over, developmental process and death. It occurs as a homeostatic mechanism. Different cells respond to different stimuli and conditions that trigger apoptosis to take place. For instance, activated plasmin from plasminogen by fibroblasts induces fibronectin proteolysis and fibroblast induces apoptosis in humans. Transforming growth factor TGF- $\beta 1$ protects fibroblasts from apoptosis induced by plasminogen activator. This is associated with the upregulation of plasminogen activator 1 (PAI-1) expression and inhabitation of plasminogen inactivation. Apoptosis plays important roles in normal physiology of mammals. So any defects in apoptosis regulation can be associated to pathogenesis of multiple diseases in mammals. So its study becomes essential. Proteins involved in apoptosis seem to contain evolutionary conserved domains as per the study. These have been observed to serve as signatures for identification, permitting applications of bioinformatics techniques to analyze various families of apoptosis regulatory proteins [15-17]. Apoptosis requires signal transduction pathway and protein-protein interactions, which is mediated by either intrinsic or extrinsic pathways. On linking of these two pathways, mitochondrial proteins are released via protein-protein interactions which trigger caspase cascade. Cancer cells can evade apoptosis. Cytotoxic drugs or anti-cancer treatment mediate cell death by activating key elements of apoptosis [18]. Mitochondrial fission and fusion is mediated by molecular machinery to form a network. During release of cytochrome $c$ and prior to caspase activation, the network disintegrates during apoptosis. Block of this fission cytochrome $\mathrm{c}$ released, is inhibited and cell death is delayed. Also according to studies, the mitochondrial antiviral signalling adaptor, MAVS is important for host defence against viral infection by inducing type 1 interferons (IFN-1). MAVS mediate viral induced apoptosis and identifies viral proteins as inhibitors of host response [19-20]. Polyamines also play a crucial role in apoptosis. Orrnothine decarboxylase (ODC) regulates intracellular polyamine synthesis. However, $\alpha$ difluromethyloornithine (DFMO) inhibits it by providing resistance to apoptosis. Also ODC activity and polyamine levels are tightly regulated. During apoptosis, ODC activity is high. And apoptosis is prevented by polyamine synthesis. Thus, inhibition of polyamine synthesis modulates epidermal growth factor receptor (EFGR) which leads to inactivation of antiapoptotic signalling cascade [21]. Acquired immune deficiency syndrome associated dementia complex (ADC) is observed mostly in Human Immunodeficiency Virus (HIV) positive patients in late phase where neuronal apoptosis occurs. This can take place through an indirect mechanism where HIV-1 infects macrophages and leukocytes. In ADC, infected microloglial cells release viral proteins which cause neuronal apoptosis [22].

\begin{abstract}
Autophagy
Autophagy adjusts cellular biomass, develops immune system and can directly control intracellular microbes as a cell autonomous innate defence. Eukaryotic pathogens organize their own autophagic machinery which is observed to contribute to microbial pathogenesis. Thus through evolution, microbes have adapted against autophagy. Matrix detachment also regulates autophagy. Autophagy is induced by detachment in both non-tumorigenic and primary epithelial cells. Depletion of autophagy regulators inhibits detachment induced autophagy. Autopagy promotes epithelial cell survival during anoikis (extracellular matrix deprivation), including detached cells harbouring antiapoptotic lesions [23, 24]. Autophagy is a lysosomal degradation pathway essential for survival, differentiation, development and homeostasis. It helps in protecting organisms against infections,
\end{abstract}


cancer, neurodegeneration, aging and heart disease. Also, fasting activates self digestion of cells by autophagy which is a evolutionary conserved process [25]. Tumour suppressor p53 activation also can stimulate autophagy. Enhanced autophagy improves the survival of p53 deficient cancer cells under conditions of hypoxia and nutrient depletion maintaining high ATP levels. Also research indicated p53 inhibition prevented activation of autophagy and providing a link between signalling pathway and autophagy to cancer associated dysregulation of p53. A pathway, only in tumour cells, can be activated only by the Fas associated death domain (FADD) of the adaptor protein. This is distant from known mechanism of FADD induced apoptosis through caspase and a physiological signal can kill normal epithelial cells through endogenous FADD protein using novel FADD death domain pathway, which activates both apoptosis and autophagy [26, 27]. Targeting TRAIL receptors has been used as a promising treatment for cancer due to preferential apoptotic susceptibility of tumour cells over normal cells to TRAIL. For tumors unresponsive to TRAIL treatment, apoptotic agents are used in combination with TRAIL to sensitive tumour cells to TRAIL mediated apoptosis. When various apoptosis defects block TRAIL mediated cell death, there is a shift of signalling cascade from default apoptosis towards cytoprotective autophagy. Apoptosis and autophagy are thus genetically regulated and evolutionarily conserved processes regulating cell fate. Both are important in development. Hence the regulation of these different processes is intimately connected and same regulators can sometimes control both apoptosis and autophagy. Under nutrient deprivation, autophagy can suppress cell death. Autophagy prevents apoptotic default pathway from being activated. Inhibition of apoptosis can lead to autophagic cell death while prevention of autophagy can precipitate death [28-30].

\section{Protein Misfolding and Cancer}

The primary etiological agent for lung cancer is cigarette smoke. A defensive role for the unfolded protein response (UPR) programme is activated due to Endoplasmic Reticulum (ER) stress on exposure to cigarette smoke which increases expression of several genes with significant roles in attenuating oxidative stress. Also several major UPR regulators increase in expression (i.e. BiP and elF2 $\alpha$ ) or phosphorylation (i.e., phosphor-elF2 $\alpha$ ) in human lung cancers. Upregulation of UPR regulators may provide a pro-survival advantage by increasing resistance to cytotoxic stresses and UPR induction could be a potential mechanism, which on attenuating, could result in efficacious treatment strategy for lung cancer [31]. Blood when touches negative surfaces like glass or plastic, it undergoes coagulation due to the activation of an enzyme cascade known as contact system. The cascade is initiated by autoactivation of Factor XII and leads to coagulation and an inflammatory response. Using patient blood and isolated proteins, Factor XII activators are identified. Factor XII gets activated by misfolded protein aggregates formed by denaturations or surface absorption, which specifically lead to activation of kallikrein-kinin system without inducing coagulation. E.g., In amyloidosis, Factor XII gets activated and kallikrein forms in blood due to deposition of misfolded plasma proteins [32]. p53 tumour suppressor is a tetrameric transcriptional enhancer whose activity is compromised by mutations that causes a substitution in its tetramerization domain. The tetramerization domain of $p 53$ is essential for efficient sitespecific DNA binding and to activate transcription from natural promoters. The biochemical and biophysical properties of peptides corresponding to amino acids of wild type human p53, include the tetramerization domain and that of a cancer derived mutant with valine substituted for glycine 334. This G334V peptide forms amyloid fibrils and hetero-oligomers with a wild type peptide. A new mechanism by which mutations inactivate p53 tumour suppressor causing etiology of many cancers compromises tetramer formation. Wildtype p53, as well as tumorigenic mutants, spontaneously lose DNA binding activity at $37^{\circ} \mathrm{C}$. This happens because folding kinetics do not change from $5^{\circ} \mathrm{C}$ to $35^{\circ} \mathrm{C}$. (DNA binding domain) DBD therefore folds by the same two-channel mechanism at physiological temperature. Unfolding rates, however, accelerate by $10,000-$ fold. So, the presence of these misfolded species, aggregate in vitro and may be degraded in the cell, leading to p53 inactivation [33, 34]. Heat shock factor 1 (HSF1) is a master regulator of heat shock response in eukaryotes. Research proves its function increases survival. It protects mice from tumours induced by mutations in p53 done in an experiment. Human cancer lines of diverse origins show much greater dependence on HSF1 function to maintain proliferation and survival than their nontransformed counterparts. HSPs are molecular chaperones that guard against promiscuous protein interactions. They facilitate normal folding of proteins and prevent misfolding and aggregation. When misfolding exceeds a certain threshold, other HSPs disaggregate proteins and refold them for destruction [35]. The androgen receptor (AR) is a ligand-activated transcription factor that is central to androgen dependent development and diseases. The activity is influenced by the length of CAG / glutamine tract in its N-terminal transactivating domain. Studies indicate expansion of this tract caused neurodegenerative disorder in men, while shorter length tracts 
caused prostrate cancer. On modelling this in mice, variations in CAG tract length within the range of normal human alleles shows occurrence of prostrate cancer. This provides insights of mechanisms by which variations in CAG / glutamine tract length influences the occurrence of human diseases [36]. The need to identify "housekeeping" markers, whose expression is stable in various experimental and clinical conditions, is of utmost clinical relevance in quantitative studies. Beta-actin (ACTB) and heat shock protein 60 (HSP60), can be regarded as reliable reference moieties for normalisation of gene and protein expression in clinical research employing human hepatic tissues [37]. AMPactivated protein kinase (AMPK) acts as a cellular fuel gauge that ensures energyconsuming processes proceed only if there are sufficient metabolic resources. Malfunction of the AMPK pathway may allow cancer cells to undergo uncontrolled proliferation irrespective of their molecular energy levels. Immunohistochemistry confirms that AMPK is dysfunctional in primary breast cancer and AMPK re-activation could have therapeutic potential in breast cancer [38]. Intrinsically disordered proteins are emerging as substantial functional constituents of mammalian proteomes. C/EBP homologous protein (CHOP) is a proto-oncogene which is an inhibitor of C/EBPs and a transcriptional activator of Activating Protein-1. CHOP inhibits of Wnt/TCF signaling and stimulation of c-Jun and sucrase-isomaltase reporter activity in intestinal colon cancer cells [39]. B lymphocyte differentiation is coordinated with the induction of high-level Ig secretion and expansion of the secretory pathway. Due to accumulation of unfolded proteins in the lumen of the ER, cells activate an intracellular signaling pathway termed as the unfolded protein response (UPR). Specific requirements of the IRE1amediated UPR subpathway are essential in the early and late stages of B lymphopoiesis [40].

\section{Protein Misfolding and Neurodegeneration}

A broad range of protein misfolding diseases arise from failure of a specific protein or peptide to adopt its native functional conformational state. This results in loss of function and gain of toxic functions. Aggregation and subsequent development of protein deposition diseases is caused mainly due to conformational changes in amyloidogenic proteins. The study showed protein fibrillogenesis occurred due to formation of unfolded amyloidogenic conformation of proteins. For ordered proteins to fibrillate, its unique structure has to be destabilized and partially unfolded whereas fibrillogenesis of natively unfolded protein involves partial folding of proteins rather than unfolding [41]. Atherosclerosis and Alzheimer disease share many risk factors including hypercholesterolemia and inflammation. Chronic inflammatory metabolites are known to link Alzheimer's disease $(A D)$ and atherosclerosis. Products of cholesterol ozonolysis have been reported in human brains. These products and related lipid derived aldehyde covalently modify $A \beta$ (Amyloid Beta protein) peptides were found to link to the pathology of $A D$. $A \beta$ misfolding occurs when soluble, monomeric oligomers first transform into spherical assemblies, then intermediates and lastly into fibrillar cross $\beta$-sheet quarternary structures known as amyloid. Polymerization of amyloid fibrils and related structures form $A B$ aggregates. The aggregation leads to neurotoxicity and inflammation, which leads to a vicious cycle of $A D$ pathology [42]. Another disease called Huntington's disease (HD) is a fatal neurodegenerative condition caused by expansion of the polyglutamine tract in the huntingtin $(\mathrm{Htt})$ protein. Genetic modifiers of HD neurodegeneration can be enriched among $\mathrm{Htt}$ protein interactors. They behave as genetic modifiers of neurodegeneration according to studies. For this, high-throughput screening for protein interactions combined with genetic validation in model organisms is used as a powerful approach for identifying novel candidate modifiers of polyglutamine toxicity. Proteins in disease datasets possess enhanced intrinsic unstructuredness. Most of these disordered proteins in the disease datasets are found to be involved in neuronal activities and have more number of interactors. Disorder prediction tools have been applied to datasets comprising of proteins involved in Huntington Disease (HD), Parkinson's disease (PD) and Alzheimer's disease (AD) [43, 44]. During acute stress in the endoplasmic reticulum (ER), mammalian prion protein $(\mathrm{PrP})$ is temporarily prevented from translocation into the ER and is routed directly for cytosolic degradation. This 'pre-emptive' quality control ( $\mathrm{pQC}$ ) system minimizes PrP aggregation in the secretory pathway during ER stress. Another case involving changes related to cellular pathway is of $t s 1$ which is a temperaturesensitive mutant of Moloney murine leukemia virus that induces a rapid spongiform encephalopathy in newborn infected mice. Perturbation of the ubiquitin-proteasome pathway might affect ts1-mediated neurodegeneration. The sensitivity of retroviral replication to the partial disruption of ubiquitin-mediated proteolysis in vivo, may have a therapeutic potential $[45,46]$. Oxidative damage to protein-coding RNA or noncoding RNA causes errors in proteins or dysregulation of gene expression. Oxidative RNA damage has been described in several neurodegenerative diseases including Alzheimer disease, Parkinson disease, etc. Oxidative RNA damage and its consequences may provide significant insights into the pathogenesis of neurodegenerative and other degenerative 
diseases and lead to better therapeutic strategies [47]. Alpha-synuclein (aSyn) misfolding is associated with several neurodegenerative disorders, including Parkinson's disease (PD). In yeast cells and in neurons aSyn accumulation is cytotoxic. A block in endoplasmic reticulum (ER)to-Golgi vesicular trafficking follows aSyn expression. The Rab guanosine triphosphatase Ypt1p, is associated with cytoplasmic aSyn inclusions whose elevated expressions protect against aSyn-induced dopaminergic neuron loss observed in animal models of PD [48]. A major step towards the understanding of mechanisms in neuronal degeneration is the identification of genes, responsible for familial variants of neurodegenerative diseases. Through studies, these disease-associated genes indicate the two faces of protein misfolding in neurodegeneration: a gain of toxic function and a loss of physiological function, or it can even occur in combination. These two faces of protein misfolding contribute to the pathomechanisms of Alzheimer's disease, frontotemporal lobar degeneration, Parkinson's disease and prion diseases. Neurodegenerative disorders are also caused by polyglutamine (polyQ) expansion in various disease proteins. Even though these polyglutamine proteins have different functions and are localized in different subcellular regions, all the polyQ diseases posses a common pathological feature: the nuclear accumulation of polyQ disease proteins and the formation of inclusions. The nuclear accumulation of polyQ proteins then leads to gene transcriptional dysregulation and neuropathology $[49,50]$.

\section{Protein Therapeutics for Immunologic and Inflammatory Diseases}

Pemphigus and pemphigoid are autoimmune skin blistering diseases. IgG was used in murine models of above diseases to test the hypothesis that the effect of Intravenous Ig (IVIG) in autoantibody-mediated skin diseases is to accelerate degradation of pathogenic IgG by saturation of neonatal $\mathrm{Fc}$ Receptor (FcRN). Circulating levels of pathogenic IgG in FcRN deficient mice were reduced than those in wild type. High dose human Ig in wild type mice prevents blistering but not in FcRN deficient mice was revealed in the study [51]. The chemokine receptor CCRS is required by many strains of HIV to enter cells. Chimeric peptides can be immunized to generate a strong antibody response focused on a short fragment of $\mathrm{N}$ terminal of CCR5 [52]. T-cells are highly sensitive to cytotoxic T-cell responses. A novel bead based approach is used to modulate T-cell response. Killer artificial APCs (KAAPCs) on coupling with apoptosis inducing a-FAS (CD95) $\operatorname{lgMmAb}$ together with HLA-A $\lg$ molecules onto beads controls $T$-cell mediated immune response. A model of T-cell mediated systematic autoimmune disorder indicated cytokine as interlukin (IL) -17 while interferon (IFN) $-\mathrm{y}$ from Th 1 cells had a protective function. Regulatory T-cells thus favour IL-17 production but also prevent the disease when administered early in the course by suppressing T-cell expansion. This indicates therapeutic capacity of T-cells [53, 54]. A study showed peptide applications to autoimmune encephalomyelitis (EAE) mice lead to anaphylaxis. So an altered peptide ligand (APL) can be engineered to prevent anaphylaxis and giving complete protection from EAE [55]. Junctional adhesion molecule-C (JAM-C) is an adhesion molecule involved in transendothelial migration of leukocytes. It is expressed by synovial fibroblasts in the lining layer in human and mouse arthritic synovial tissue. Treatment of mice with an anti JAM-C antibody significantly reduced the serenity of Antigen Induced Arthritis under research [56]. Further, the expression and regulation of four major inflammatory cytokines (MIP-2, KC, IL-1 $\beta, I L-61$ ) is important in Fungal Keratitis (FK). Of them, high level expression of MIP-2 and IL-1 $\beta$ is an important and major factor in the corneal pathogenesis. Specific polyclonal antibodies are administered to inhibit chemokines and cytokines for relieving injury by FK [57]. Macrophages were found to be mainly responsible for acute inflammatory diseases. Microparticles made from aliphatic polyketals are greatly used in treating inflammatory diseases. Since some are unsuitable a polyketal PK3 is developed by encapsulating with an antiinflammatory drug imatinib. As a result, PK3 improved efficacy of imatinib in treating acute liver failure [58]. Neurogenic inflammation in mice was assessed by pruritogenic response induced by trypsin. Trypsin intradermal injection serves as a reproducible model for the study of itching and involvement of PAR-2 receptors. Trypsin induced itching, depends on neurogenic inflammation with TRPVI receptors role [59]. IL-32, a new cytokine in human found from studies, is an invitro producer of tumour necrosis factor alpha (TNFa). TNFa induces IL-32 mRNA as a result relation between them is studied easily. IL-32 is closely associated with TNFa and contributes to exacerbation of TNFa related inflammatory arthritis and colitis [60].

\section{References}

[1] Janin J., Bahadur R.P., Chakrabarti P. (2008) Q Rev Biophys 41 (2):133-80.

[2] Cho K.I., Lee K., Lee K.H., Kim D., Lee D. (2006) Proteins 65(3): 593-606.

[3] Teichmann S.A. (2002) Bioinformatics Vol. 18 no. 90002 Pages S249.

[4] Keskin O., Tuncbag N., Gursoy A. (2008) Curr Pharm Biotechnol; 9(2): 67-76.

[5] Aung Z., Tan S.H., Ng S.K., Tan K.L. (2007) Comput Syst Bioinformatics Conf.; 6: 287-97. 
[6] Lee H., Deng M., Sun F., Chen T. (2006) BMC Bioinformatics; 7:269.

[7] Fossum E., Friedel C.C., Rajagopala S.V., Titz B., Baiker A., Schmidt T., Kraus T., Stellberger T., Rutenberg C., Suthram S., Bandyopadhyay S., Rose D., von Brunn A., Uhlmann M., Zeretzke C., Dong Y.A., Boulet H., Koegl M., Bailer S.M., Koszinowski U., Ideker T., Uetz P., Zimmer R., Haas J. (2009) PLoS Pathog.; 5(9):e1000570.

[8] Dixon D.A., Tolley N.D., Bemis S. K., Martinez M.L., Weyrich A.S., Morrow J.D., Prescott S.M., Zimmerman G.A. (2006) J Clin Invest; 116(10):2727-38.

[9] McDonald D., Waterbury L., Knight R., Betterton M.D. (2008) Biol Direct; 3:49.

[10] Bortoluzzi S., Romualdi C., Bisognin A., Danieli G.A. (2003) Physiol Genomics. vol (3) 223-7.

[11] Davé V., Childs T., Xu Y., Ikegami M., Besnard V., Maeda Y., Wert S.E., Neilson J.R., Crabtree G.R., Whitsett J.A. (2006) J Clin Invest.; 116(10):2597609.

[12] Kim H.J., Martemyanov K.A., Thayer S.A. (2008) J Neurosci.; 28(48):12604-13.

[13] Dezso Z., Nikolsky Y., Nikolskaya T., Miller J., Cherba D., Webb C., Bugrim A. (2009) BMC Syst Biol. 23;3:36.

[14] Linding R., Jensen L.J., Ostheimer G.J., van Vugt M.A., Jørgensen C., Miron I.M., Diella F., Colwill K., Taylor L., Elder K., Metalnikov P., Nguyen V., Pasculescu A., Jin J., Park J.G., Samson L.D., Woodgett J.R., Russell R.B., Bork P., Yaffe M.B., Pawson T. (2007) Cell. 129(7):1415-26.

[15] Elmore S. Apoptosis: (2007) Toxicol Pathol.; 35(4):495-516.

[16] Horowitz J.C., Rogers D.S., Simon R.H., Sisson T.H., Thannickal V.J. (2008) $A m$ J Respir Cell Mol Biol.; 38(1):78-87.

[17] John Reed J.C., Doctor K., Rojas A., Zapata J.M., Stehlik C., Fiorentino L., Damiano J., Roth W., Matsuzawa S., Newman R., Takayama S., Marusawa H., Xu F., Salvesen G., Godzik A. RIKEN GER Group; (2003) GSL Members, Genome Res. 13(6B):137688.

[18] Chu L.H., Chen B.S. (2008) BMC Syst Biol. 2:56.

[19] Suen D.F., Norris K.L., Youle R.J. (2008) Genes Dev.; 22(12):1577-90.

[20] Lei Y., Moore C.B., Liesman R.M., O'Connor B.P., Bergstralh D.T., Chen Z.J., Pickles R.J., Ting J.P. (2009) PLoS One.; 4(5):e5466.

[21] Ray R.M., Bhattacharya S., Johnson L.R. (2007) Cell Signal.; 19(12):2519-27.
[22] Bachis A., Biggio F., Major E.O., Mocchetti I. (2009) J Neuroimmune Pharmacol.; 4(1):150-60.

[23] Deretic V., Levine B. (2009) Autophagy, 5(6):527-49.

[24] Fung C., Lock R., Gao S., Salas E., Debnath J. (2008) Mol Biol Cell; 19(3):797-806.

[25] Levine B., Kroemer G. (2008) Cell; 132(1):27-42.

[26] Tasdemir E., Maiuri M.C., Galluzzi L., Vitale I., Djavaheri-Mergny M., D'Amelio M., Criollo A., Morselli E., Zhu C., Harper F., Nannmark U., Samara C., Pinton P., Vicencio J.M., Carnuccio R., Moll U.M., Madeo F., Paterlini-Brechot P., Rizzuto R., Szabadkai G., Pierron G., Blomgren K., Tavernarakis N., Codogno P., Cecconi F., Kroemer G. (2008) Nat Cell Biol.; 10(6):676-87.

[27] Thorburn J., Moore F., Rao A., Barclay W.W., Thomas L.R., Grant K.W., Cramer S.D., Thorburn A. (2005) $\mathrm{Mol}$ Biol Cell. 16(3):1189-99.

[28] Han J., Hou W., Goldstein L.A., Lu C., Stolz D.B., Yin X.M., Rabinowich H. (2008) J Biol Chem.; 283(28):19665-77.

[29] Thorburn A. (2008) Apoptosis.; 13(1):1-9

[30] Boya P., González-Polo R.A., Casares N., Perfettini J.L., Dessen P., Larochette N., Métivier D., Meley D., Souquere S., Yoshimori T., Pierron G., Codogno P., Kroemer G. (2005) Mol Cell Biol.; 25(3):1025-40.

[31] Jorgensen E., Stinson A., Shan L., Yang J., Gietl D., Albino A.P. (2008) BMC Cancer, 8:229.

[32] Maas C., Govers-Riemslag J.W., Bouma B., Schiks B., Hazenberg B.P., Lokhorst H.M., Hammarström P., ten Cate H., de Groot P.G., Bouma B.N., Gebbink M.F. J Clin Invest.; 118(9):3208-18.

[33] Higashimoto Y., Asanomi Y., Takakusagi S., Lewis M.S., Uosaki K., Durell S.R., Anderson C.W., Appella E., Sakaguchi K. (2006) Biochemistry.; 45(6):1608-19.

[34] Butler J.S., Loh S.N., (2006) Protein Sci. (11):2457-65.

[35] Dai C., Whitesell L., Rogers A.B., Lindquist S., (2007) Cell; 130(6):1005-18.

[36] Lieberman A.P., Robins D.M., (2008) J Alzheimers Dis.; 14(2):247-55.

[37] Sun S., Yi X., Poon R.T., Yeung C., Day P.J., Luk J.M. (2009) BMC Cancer.; 9:309.

[38] Hadad S.M., Baker L., Quinlan P.R., Robertson K.E., Bray S.E., Thomson G., Kellock D., Jordan L.B., Purdie C.A., Hardie D.G., Fleming S., Thompson A.M. (2009) BMC Cancer.; 9:307. 
[39] Singh V.K., Pacheco I., Uversky V.N., Smith S.P., MacLeod R.J., Jia Z., (2008) J Mol Biol.; 380(2):313-26.

[40] Zhang K., Wong H.N., Song B., Miller C.N., Scheuner D., Kaufman R.J. (2005) J Clin Invest:; 115(2):268-81.

[41] Uversky V.N. (2008) Curr Alzheimer Res.; 5(3):260-87.

[42] Zhang Q., Powers E.T., Nieva J., Huff M.E., Dendle M.A., Bieschke J., Glabe C.G., Eschenmoser A., Wentworth P. Jr., Lerner R.A., Kelly J.W. (2004) Proc Natl Acad Sci U S A; 101(14):4752-7.

[43] Kaltenbach L.S., Romero E., Becklin R.R., Chettier R., Bell R., Phansalkar A., Strand A., Torcassi C., Savage J., Hurlburt A., Cha G.H., Ukani L., Chepanoske C.L., Zhen Y., Sahasrabudhe S., Olson J., Kurschner C., Ellerby L.M., Peltier J.M., Botas J., Hughes R.E. (2007) PLoS Genet; 3(5):e82.

[44] Raychaudhuri S., Dey S., Bhattacharyya N.P., Mukhopadhyay D. (2009) PLoS One.; 4(5):e5566.

[45] Rane N.S., Kang S.W., Chakrabarti O., Feigenbaum L., Hegde R.S. (2008) Dev Cell.; 15(3):359-70.

[46] Mei Z. M., Thurig S., Tsirigotis M., Wong P.K., Reuhl K.R., Gray DA. (2003) J Virol; 77(13):7193-201.

[47] Castellani R.J., Nunomura A., Rolston R.K., Moreira P.I., Takeda A., Perry G., Smith MA. (2008) Int J Mol Sci.; 9(5):789-806.

[48] Cooper A.A., Gitler A.D., Cashikar A., Haynes C.M., Hill K.J., Bhullar B., Liu K., Xu K., Strathearn K.E., Liu F., Cao S., Caldwell K.A., Caldwell G.A., Marsischky G., Kolodner R.D., Labaer J., Rochet J.C., Bonini N.M., Lindquist S. (2006) Science; 313(5785):324-8.

[49] Winklhofer K.F., Tatzelt J., Haass C. (2008) EMBO J.; 27(2):336-49.

[50] Havel LS., Li S., Li X.J. (2009) Mol Brain. 2(1):21.

[51] Li N., Zhao M., Hilario-Vargas J., Prisayanh P., Warren S., Diaz L.A., Roopenian D.C., Liu Z. (2005) J Clin Invest:;115(12):3440-50.

[52] Chain B.M., Noursadeghi M. (2005) J Clin Invest.; 115(12):3440-50.

[53] Schütz C., Fleck M., Mackensen A., Zoso A., Halbritter D., Schneck J.P., Oelke M., (2008) Blood:; 111(7):3546-52.

[54] Lohr J., Knoechel B., Wang J.J., Villarino A.V., Abbas A.K. (2006) J Exp Med.; 203(13):2785-91.

[55] Leech M.D. Chung C.Y., Culshaw A., Anderton S.M. (2007) Eur J Immunol.; 37(12):3576-81.

[56] Palmer G., Busso N., Aurrand-Lions M., Talabot-Ayer D., Chobaz-Péclat V.,
Zimmerli C., Hammel P., Imhof B.A., Gabay C. (2007) Arthritis Res Ther.; 9(4):R65.

[57] Zhong W., Yin H., Xie L. (2009) Mol Vis.; 15:1303-11.

[58] Yang S.C., Bhide M, Crispe I.N., Pierce R.H., Murthy N. (2008) Bioconjug Chem.; 19(6):1164-9.

[59] Costa R., Marotta D.M., Manjavachi M.N., Fernandes E.S., Lima-Garcia J.F., Paszcuk A.F., Quintão N.L., Juliano L., Brain S.D., Calixto J.B. (2008) $\mathrm{Br} J$ Pharmacol.; 154(5):1094-103.

[60] Shoda H., Fujio K., Yamaguchi Y., Okamoto A., Sawada T., Kochi Y., Yamamoto K. (2006) Arthritis Res Ther.; 8(6):R166. 